\title{
A Method for the Measurement of Mercury in Human Whole Blood
}

\author{
Alicia E. Stube, Helene H. Freiser, Charles R. Santerre* \\ Department of Nutrition Science, Purdue University, West Lafayette, Indiana, USA \\ E-mail: ${ }^{*}$ santerre@purdue.edu \\ Received July 26, 2011; revised September 1, 2011; accepted September 12, 2011
}

\begin{abstract}
A method for measuring total mercury in human whole blood using Thermal Decomposition-Amalgamation/ Atomic Absorption Spectrophotometry (TDA/AAS) was developed and applied to a study of women that were fish consumers. This method has a limit of detection of $0.33 \mu \mathrm{g} / \mathrm{L}$. The blood mercury concentrations measured ranged from $0.74 \mu \mathrm{g} / \mathrm{L}$ to $14.80 \mu \mathrm{g} / \mathrm{L}$, with a mean of $3.36 \mu \mathrm{g} / \mathrm{L}$. Accuracy was within $15 \%$ of the expected value at the lower concentrations and within $10 \%$ at higher concentrations. Some 560 analysis were completed in about three weeks and the mean error in precision was $1.8 \%$ when measured in duplicate. It was concluded that this method is viable for use in clinical settings, with the benefit of small sample volumes and minimal sample preparation.
\end{abstract}

Keywords: Mercury, TDA/AAS, Human Blood

\section{Introduction}

Mercury (Hg) status can be assessed by hair or blood biomarkers [1]. Humans who consume certain types of fish may have higher mercury concentrations in their system [2]. Methyl mercury is readily absorbed from the gut into the bloodstream, where it binds to red blood cells [3]. Previous analytical methods for measuring blood mercury tested only red blood cells; however whole blood methods are now gaining ground [4]. Use of whole blood reduces sample preparation time and eliminates uncertainty relating to cell number. Centrifugation and separation of red blood cells also present an opportunity for analyte loss and increases the overall uncertainty in measurement. Reducing the time and steps involved in sample preparation facilitates measurement of mercury in clinical settings.

The traditional methods for measuring mercury are cold-vapor atomic absorption spectroscopy (CVAAS) and inductively-coupled plasma mass spectrometry (ICP-MS) $[5,6]$. These methods are accepted as reliable and sensitive; however, throughput of samples is slow, with high equipment and reagent costs. The samples are exposed to concentrated acids and extended heating, which increase analyte loss [7]. Thermal DecompositionAmalgamation/Atomic Absorption Spectrophotometry (TDA/AAS) is an emerging technique for rapidly testing large numbers of samples [8]. Samples are introduced directly to the instrument and analyzed in minutes without the need for extensive preparation [9]. This high throughput method allows large number of samples to be analyzed accurately and rapidly with less analyte loss than the conventional methods listed above. TDA/AAS also contributes to the movement towards green chemistry, requiring no preparatory reagents or hazardous chemicals. This technology has already been utilized for analysis of avian blood samples with high mercury concentrations [4]; however, because of the low concentration of mercury in human blood there is currently no reliable TDA/AAS method. This paper proposes a method for TDA/AAS analysis of whole blood that would be usable for clinical applications with subjects within typical human blood mercury concentrations.

\section{Materials and Methods}

\subsection{Instrumentation and Chemicals}

The Tricell DMA 80.3 Direct Mercury Analyzer (Milestone, Inc., Shelton, CT) was utilized for mercury measurements. Quartz sample boats of $1.5 \mathrm{ml}$ volume were obtained from the same vendor. The auto-sampler of the DMA 80.3 instrument had forty slots that could be filled with sample boats. A stock mercury solution (Accustan- 
dard, New Haven, CT) of $0.1 \mu \mathrm{g} / \mathrm{ml}$ mercury in $5 \%$ nitric acid was used to calibrate the instrument and act as a running standard. SRM 966 (NIST, Gaithersburg, MD), Toxic Elements in Bovine Blood, level 1, was used as Standard Reference Material (SRM). A single human blood sample from an individual low in mercury was used to establish a baseline and for spiking. For verification, four samples of blood from 70 subjects were utilized, for a total of 280 samples. Blood was drawn into EDTA $\mathrm{K}_{3}$ tubes (Greiner BioOne, Monroe, NC). These samples were stored frozen at $-80^{\circ} \mathrm{C}$ and then thawed and subjected to vortex mixing for at least 30 seconds immediately prior to analysis.

Approval for human subject research was obtained from the Purdue University Institutional Review Board, Research Project Number 0709005855. All subjects signed a consent form detailing the research procedures and any possible risks that might be incurred as a result of participation.

\subsection{Calibration}

Cells 0 and 1 of the DMA 80.3 were calibrated using different volumes of the standard mercury solution in quartz boats (i.e. at 0.05, 0.1, 0.2, 0.3, 0.5, 1, 2, 3, 5, $7 \mathrm{ng}$ of mercury) for Cell 0 . That range was extended to $10 \mathrm{ng}$ of mercury for Cell 1. Cell 2 was not calibrated as its measurement range was well above the expected mercury levels in human blood. The linearity of the calibration curve was evaluated using this dataset and a best fit model was established using the DMA 80.3's internal software. An S-curve was used to fit the data, resulting in a correlation equation of

$$
\begin{aligned}
A= & -0.0003 *[\mathrm{Hg}]^{3}+0.0015 *[\mathrm{Hg}]^{2} \\
& +0.0734 *[\mathrm{Hg}]-0.0013
\end{aligned}
$$

and a coefficient of correlation, $\mathrm{R}^{2}$, of 1.00. An injection of $20 \mu \mathrm{L}$ of $100 \mu \mathrm{g} / \mathrm{L}$ standard was used to confirm the calibration and was measured within $2 \%$ of the expected value. The limit of detection with this calibration was $0.33 \mu \mathrm{g} / \mathrm{L}$ for a $150 \mu \mathrm{L}$ blood sample with $0.0495 \mathrm{ng}$ mercury. Below $0.33 \mu \mathrm{g} / \mathrm{L}$, measurements became imprecise due to background noise and residual mercury on the boats.

The calibration was confirmed daily at the start of each run using a $20 \mu \mathrm{L}$ injection of standard solution containing $2 \mathrm{ng}$ mercury. If the verification sample was off by more than $5 \%$, a second 2 ng of mercury sample was run to establish a calibration factor to be applied to all of that day's measurements. If the verification was off by more than $10 \%$, a new calibration curve was established. The standard solution was used in conjunction with a $150 \mu \mathrm{L}$ injection of SRM 966 level 1 to ensure interday repeatability.

\subsection{Instrument Parameters}

Various parameters were tested using human blood, until a method which provided acceptable recovery and precision was found. These parameters included changes to sample volume and drying/decomposition conditions. EPA method 7473 was used as a starting point, as it had previously been validated as an effective and reliable method to measure mercury in fish tissues [10]. A method provided by the manufacturer was also considered, but found unsuitable for whole blood analysis. With larger number of samples, this method resulted in residue accumulation in the catalyst tube and cells, wearing down the mercury vapor lamp and ultimately resulting in greater uncertainty between duplicate samples.

The optimal process conditions were found to be a sample size of $150 \mu \mathrm{L}$, drying at $120^{\circ} \mathrm{C}$ for $2 \min 20 \mathrm{sec}$, 1 min ramp to $650^{\circ} \mathrm{C}$, and finally decomposition at $650^{\circ} \mathrm{C}$ for $3 \mathrm{~min} 30 \mathrm{sec}$. This set of conditions was chosen based on precision and reproducibility over a number of sampling days, as well as completeness of combustion. Sample volumes greater than $150 \mu \mathrm{L}$ tended to boil over in the oven and overwhelm the catalyst tube with an accumulation of ash. However, at smaller volumes, there was not enough absolute mercury present in the sample for reliable analysis.

Development of the drying/decomposition ramp was also important to avoid boiling the sample out of the boats too quickly. Too high an initial drying temperature would result in boats bubbling over and loss of volume. In these cases, a blackened crust was observed on the top and sides of the boat. The ramp was used to slowly heat the dried samples, avoiding volume loss. The decomposition temperature recommended by the EPA method for mercury analysis in wastewater [8] was too low to properly combust the complex matrix of whole blood and would leave blackened residue in the boats after heating. Raising the temperature to $650^{\circ} \mathrm{C}$ on the DMA 80.3 resulted in complete combustion.

The remaining instrument parameters for sample analysis included a purge time of $30 \mathrm{sec}$, the amalgam time was set to $12 \mathrm{sec}$ and the recording time measured $30 \mathrm{sec}$. Before a quartz boat could be used again after a blood analysis, it needed to be cleaned by going through one cycle at a drying temperature of $300^{\circ} \mathrm{C}$ for $1 \mathrm{~min}$ and decomposition at $650^{\circ} \mathrm{C}$ for $3 \mathrm{~min}$.

\subsection{Precision and Accuracy Study}

Two samples of human blood, one from a non-fisheater and one from a regular fisheater, were used to test the 
feasibility of the method. Once a method with less than $10 \%$ spread between triplicate samples was identified, spiked samples of the low mercury blood were used as to calculate precision and accuracy. Quartz boats were utilized for blood samples and standards; a blank in the form of an empty nickel boat was placed at the beginning and end of every sample run, as well as between each individual sample to ensure any residual mercury from the previous sample was burned off.

The baseline low mercury human blood sample, $150 \mu \mathrm{L}$ for each concentration, was spiked with fixed amounts of standard mercury solution at 2.2, 5, 10, 20 and $30 \mu \mathrm{L}$. The series of standards was analyzed five times. For each concentration, a predicted mercury concentration was also calculated, taking into account the approximate mercury content of the baseline blood. Measurements were compared to calculated concentrations, and the percent error was used as an estimate of accuracy. For precision calculations, the mean and relative standard deviation for the five samples was determined.

\subsection{Verification Study}

Human blood samples were used to check the reference range and confirm that the method was useful for analysis of a large number of human blood samples. Before and after each day's run of human blood samples, a $150 \mu \mathrm{L}$ sample of SRM 966 was analyzed, and the results were used to formulate a control chart. Quality control limits were set at two standard deviations from the mean. Runs where the SRM value fell outside the three standard deviation control limits were removed and the samples done that day re-analyzed after a recalibration. The samples for the verification study were done in duplicate. If more than $10 \%$ error was observed between duplicate samples, the samples were reanalyzed. Data from the control chart were tracked to observe between day precision.

\section{Results and Discussion}

\subsection{Assessment of Method}

Accuracy and precision were measured using spiked blood samples. Baseline blood was taken from a non-fish eater and estimated to contain $0.122 \mu \mathrm{g} / \mathrm{L}$ mercury after five sample runs. This blood was used as a baseline to represent the low anchor of the range of expected human blood mercury concentrations. Aliquots of the baseline blood were spiked at five concentrations using a standard mercury solution; the mean, standard deviation, and calculated errors in precision and accuracy are shown in Table 1.
At each concentration, the measured DMA 80.3 values were slightly lower than the expected blood mercury levels. Accuracy was within $15 \%$ of the expected value at the lower concentrations and within $10 \%$ at higher concentrations. When $2.2 \mu \mathrm{L}$ of standard solution were added (expected concentration $1.6 \mu \mathrm{g} / \mathrm{L}$ ), $87.5 \%$ of the mercury was recovered and measured by the instrument. When the spiked amount was raised to $30 \mu \mathrm{L}, 95.6 \%$ of the mercury was recovered. Loss of mercury may also be attributable to the volatility of the standard mercury solution; some of the spiked mercury may have been lost before the sample could be introduced into the instrument [7].

Error in precision was very low across all of the spiked blood samples, consistently below 5\%. This did not appear to relate to concentration, as values were similar at both high and low concentrations. Overall the blood method showed excellent recovery in spiked samples as well as intra-day precision.

\subsection{Method Validation via Analysis of Human Blood Samples}

A total of 280 different blood samples from 70 different female subjects were used to evaluate the method's feasibility in clinical applications. These measurements are shown in Figure 1.

Table 1. Precision and accuracy of spiked blood samples.

\begin{tabular}{ccccc}
\hline $\begin{array}{c}\text { Known Hg } \\
(\mu \mathrm{g} / \mathrm{L})\end{array}$ & $\begin{array}{c}\text { Measured Hg } \\
(\mu \mathrm{g} / \mathrm{L})\end{array}$ & $\begin{array}{c}\text { Std Dev } \\
(\mu \mathrm{g} / \mathrm{L})\end{array}$ & $\begin{array}{c}\text { Error in } \\
\text { Accuracy }\end{array}$ & $\begin{array}{c}\text { Error in } \\
\text { Precision }\end{array}$ \\
\hline 1.60 & 1.40 & 0.036 & $12.0 \%$ & $2.6 \%$ \\
3.45 & 3.08 & 0.047 & $10.7 \%$ & $1.5 \%$ \\
6.79 & 6.18 & 0.220 & $8.9 \%$ & $3.5 \%$ \\
13.45 & 13.00 & 0.454 & $3.7 \%$ & $3.5 \%$ \\
20.12 & 19.25 & 1.234 & $4.3 \%$ & $6.4 \%$ \\
\hline
\end{tabular}

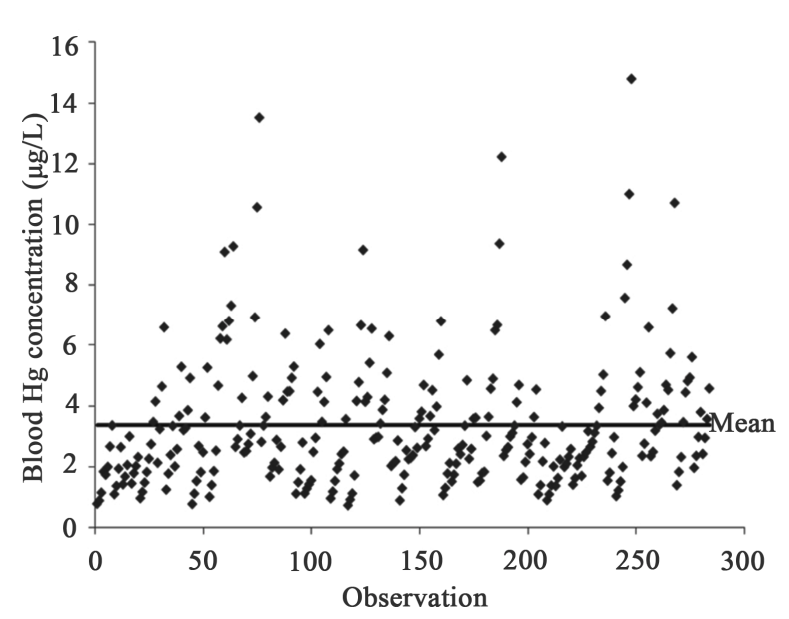

Figure 1. Blood mercury concentrations measured by TDA /AAS. Mean is represented as a solid line. 
These samples were run in duplicate and intra-sample precision was measured. Blood mercury concentrations ranged from $0.74 \mu \mathrm{g} / \mathrm{L}$ to $14.80 \mu \mathrm{g} / \mathrm{L}$, with a mean of $3.36 \mu \mathrm{g} / \mathrm{L}$ and the 50 th percentile at $2.76 \mu \mathrm{g} / \mathrm{L}$. All samples were above the detection limit and all had errors in precision of less than $10 \%$; the mean error was $1.8 \%$ when measured in duplicate.

The current EPA reference dose $(0.1 \mu \mathrm{g} / \mathrm{kg}$ body weight-day) has been shown to provide a concentration in human blood of $5.8 \mu \mathrm{g} / \mathrm{L}$ [11]. Recurring levels above this value is believed to cause harm, in particular to the developing fetus. Most of the blood samples collected in this study registered lower than that threshold, as evident from Figure 1, but some fish-eating subjects had unusually high values. For those individuals quick corrective actions might be needed, highlighting the advantage of the described method.

Each sample required about 10 minutes for analysis; when analyzed in duplicate, about twenty-four minutes was spent on the analysis of each sample. In total, the time required to measure mercury concentration in these 280 samples of blood (560 analyses) was about three weeks if samples were prepared and loaded during the 40 hour work week. If samples are prepared and loaded during nights and weekends as well, sample throughput can be increased further. Preparing the samples and filling the auto-sampler tray required less than an hour so most of the time spend for each lot consisted of unattended data collection. By comparison, it can take up to a day of preparation work to analyze samples by CVAAS or ICP-MS.

\section{Conclusions}

The method described here is a viable way to measure total mercury concentration in whole blood. Mercury in human blood can be measured reliably and accurately in less than ten minutes per sample. This method can be useful for large clinical studies with high volumes of samples to analyze. Since only a $150 \mu \mathrm{L}$ sample volume is required, it may also not be necessary to draw venous blood to measure an individual's blood mercury levels. A fingerstick with a deep puncture blade can draw up to 1 $\mathrm{mL}$ of blood, sufficient for analysis via this method. Fingersticks are faster and less invasive than venous blood draws. Collection of fingerstick samples makes measurement of blood mercury more accessible and practical for situations outside of the clinical setting. The ability to utilize small sample volumes combined with a rapid turnaround time makes measuring blood levels just as viable as hair concentrations for efficient assessment of mercury exposure. Use of this method for analysis of total mercury in human whole blood provides a green alternative that is simple, reliable and time efficient.

\section{Acknowledgements}

Financial support for this study was obtained from the US Department of Agriculture, National Institute of Food and Agriculture (No. 07-51110-03804). Collection of blood samples was done by Doug Maish, EMT-P, Purdue University.

\section{References}

[1] T. W. Clarkson and L. Magos, "The Toxicology of Mercury and Its Chemical Compounds," Critical Reviews in Toxicology, Vol. 36, No. 8, 2006, pp. 609-662. doi:10.1080/10408440600845619

[2] E. Oken, J. S. Radesky, R. O. Wright, D. C. Bellinger, C. J. Amarasiriwardena, K. P. Kleinman, H. Hu and M. W. Gillman, "Maternal Fish Intake during Pregnancy, Blood Mercury Levels, and Child Cognition at Age 3 Years in a US Cohort," American Journal of Epidemiology, Vol. 167, No. 10, 2008, pp. 1171-1191.

doi:10.1093/aje/kwn034

[3] L. W. Chang, "Toxicology of Metals," Lewis Publishers, Boca Raton, 1996, p. 1052.

[4] J. T. Ackerman, C. A. Eagles-Smith, J. Y. Takekawa, J. D. Bluso and T. L. Adelsbach," Mercury Concentrations in Blood and Feathers of Prebreeding Foster's Terns in Relation to Space Use of San Francisco Bay, California, Habitats," Environmental Toxicology and Chemistry, Vol. 27, No. 4, 2008, pp. 897-908. doi:10.1897/07-230.1

[5] APHA. 3112, "Metals by Cold-Vapor Atomic Absorption Spectrometry," In: M. A. H. Franson, Ed., Standard Methods for the Examination of Water and Wastewater, Ame- rican Public Health Association, Washington D. C. Vol. A, 1998, pp. (3-22) - (3-24).

[6] APHA. 3120, "Inductively Coupled Plasma (ICP) Method," In: M. A. H. Franson, Ed., American Public Health Association, Washington D.C., Vol. B, 1998, pp. (3-38) (3-52).

[7] D. D. Afonso, Z. Arsian and A. J. Bednar, “Assessment of Matrix-Dependent Analyte Stability and Volatility during Open-Vessel Sample Dissolution for Arsenic, Cadium, Mercury and Selenium," Microchimica Acta, Vol. 167, No. 1-2, 2009, pp. 53-59. doi:10.1007/s00604-009-0218-3

[8] EPA, "Method 7473: Mercury in Solids and Solutions by Thermal Decomposition, Amalgamation, and Atomic Adsorption Spectroscopy," Agency UEP, 1998.

[9] S. J. M. Butala, L. P. Scanlan and S. N. Chaudhuri, "A Detailed Study of Thermal Decomposition, Amalgamation/Atomic Absorption Spectrophotometry Methodology for the Quantitative Analysis of Mercury in Fish and Hair," Journal of Food Protection, Vol. 69, 2006, pp. 2720-2728. 
[10] J. A. Lasrado, C. R. Santerre, S. M. Shim and J. R. Stahl, "Analysis of Mercury in Sportfish Tissue by Thermal Decomposition, Amalgamation/Atomic Absorption Spectrophotometry," Journal of Food Protection, Vol. 68, 20 05, pp. 879-881.
[11] L. Trasande, P. J. Landrigan and C. Schechter, "Public Health and Economic Consequences of Methyl Mercury Toxicity to the developing Brain," Environmental Health Perspectives, Vol. 113, 2005, pp. 590-596. doi:10.1289/ehp.7743 